\title{
Design and Modeling of a Miniaturized Substrate Integrated Waveguide Using Embedded SRRs
}

\author{
Farhad Farzami, Student Member, IEEE, Keyvan Forooraghi, Member, IEEE, and Majid Norooziarab, \\ Student Member, IEEE
}

\begin{abstract}
A substrate integrated waveguide (SIW) loaded by embedded split ring resonators (SRRs) with transversal negative effective permeability is proposed. It is shown that the structure can support propagation of backward waves below cut off frequency. Therefore the width of the SIW structure can be considered less than half a wavelength at the cut off frequency, which means that the SIW structure is miniaturized. In this paper, design and modeling of a miniaturized SIW structure is proposed. An experimental SIW loaded with double embedded SRRs in $4.75 \mathrm{GHz}$ frequency band has been designed, fabricated and tested. The measured and simulated results show a passband for backward waves below the cutoff frequency. It is also shown that the phase at a certain frequency in the backward passband increases as the physical length of the loaded SIW is increased (opposite to forward wave propagation), which proves that backward waves propagate below the cut off frequency.
\end{abstract}

Index Terms - Substrate integrated waveguide (SIW), embedded split ring resonator (SRR), backward waves.

\section{INTRODUCTION}

$\mathrm{A}$ substrate integrated waveguide (SIW) is similar to a rectangular waveguide filled with a dielectric, except for the narrow walls which are replaced by two rows of metal cylinders (vias), Fig.1. If the distances between the vias are small enough compared to the wavelength, the propagation characteristics of the SIW can be considered as a typical metallic rectangular waveguide [1]. High Q, high power handling capability, low profile and easy fabrication, etc; are of the advantages of these structures. The most important advantage of SIWs relative to rectangular waveguides is that they can be integrated with planar structures. Considering the above feature, SIWs have been widely used in many microwave and millimeter wave devices [2].

On the other hand, split ring resonators (SRRs) which are a kind of metamaterials have been widely used in many microwave and millimeter wave components; because of their natural resonant property and that they can show negative permeability. Miniaturization of a conventional metallic rectangular waveguide can be realized when it is loaded with

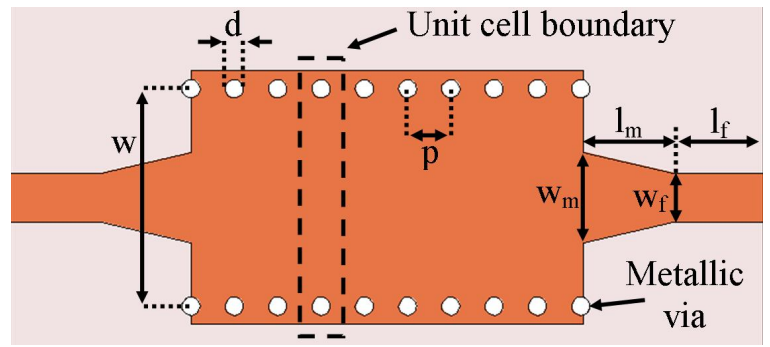

Fig. 1. Microstrip-fed SIW structure. The dimensions are $\mathrm{d}=1 \mathrm{~mm}, \mathrm{p}=2.4 \mathrm{~mm}$, $\mathrm{w}=12.2 \mathrm{~mm}, \mathrm{w}_{\mathrm{m}}=5.4 \mathrm{~mm}, \mathrm{w}_{\mathrm{f}}=2.7 \mathrm{~mm}$ and $\mathrm{l}_{\mathrm{m}}=\mathrm{l}_{\mathrm{f}}=5 \mathrm{~mm}$

SRRs [3]. However the miniaturized rectangular waveguide is three dimensional and is not suitable for planar implementations.

In this paper a split ring resonator which can be embedded in thin substrates is proposed. It is shown that an SIW can be miniaturized when it is loaded by these embedded SRRs. It is also shown that the cutoff frequency of the waveguide structure can be arbitrarily tuned if the SRRs are designed properly. An SIW structure with a cutoff frequency of $7 \mathrm{GHz}$ is designed. The structure is then loaded by double embedded SRRs to provide a passband for backward waves in $4.75 \mathrm{GHz}$. Finally the structure is fabricated and measured. The measured results are in good agreement with those obtained by simulations.

Section II discusses the SIW and the SRR structures. An SIW loaded by a negative transversal permeability embedded SRRs is designed, simulated and measured in section III. Section IV concludes the paper.

\section{WAVEGUIDE STRUCTURE}

\section{A. The SIW structure}

The geometry of a microstrip-fed SIW structure is shown in Fig. 1. Numerical methods for modeling an SIW are based on analyzing a unit cell of SIW. The boundary of a unit cell forming a periodic SIW structure is shown in Fig. 1 with dashed lines. Moreover, SIWs can be modeled as rectangular waveguides. The width of an SIW can be obtained with different methods. In [1] the relation between the equivalent width of a rectangular waveguide $\left(\mathrm{w}_{\mathrm{e}}\right)$ and the width of the 


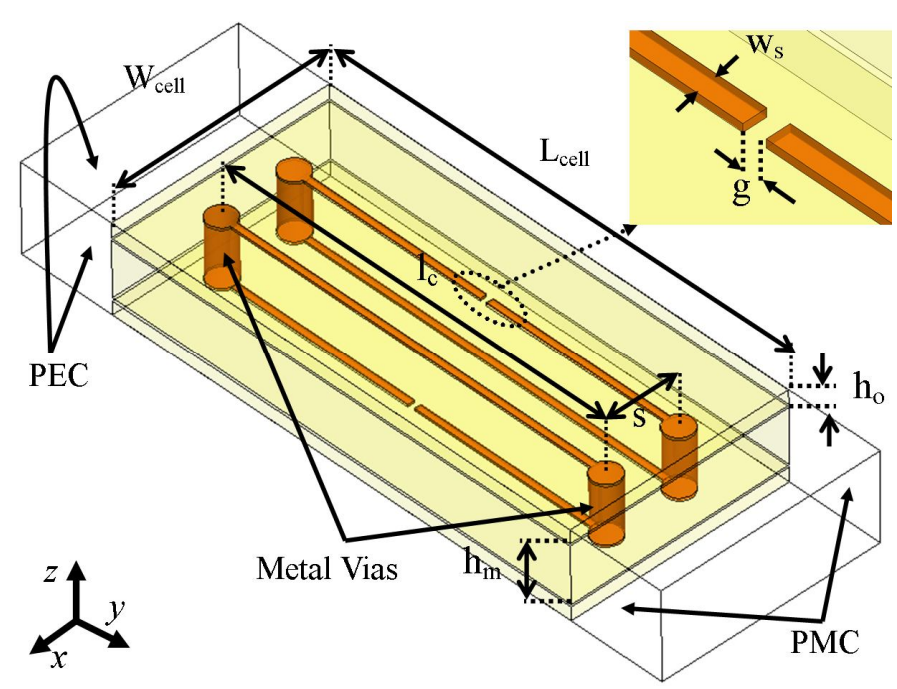

(a)

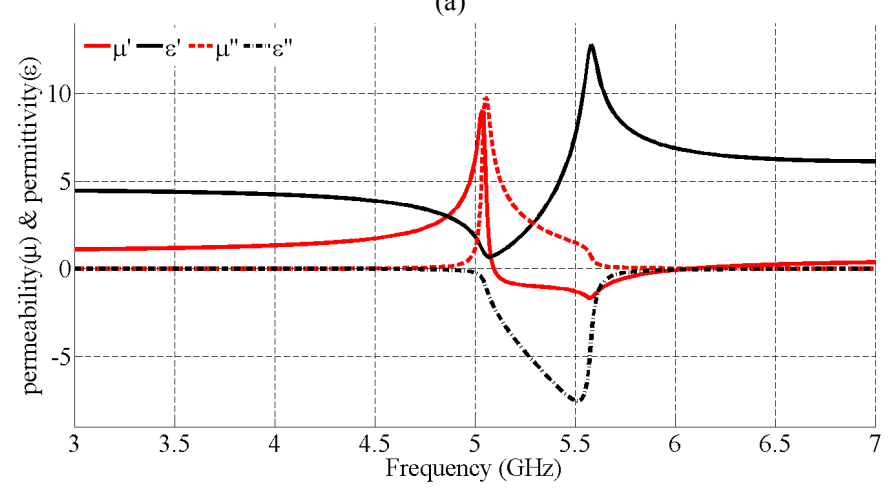

(b)

Fig. 2. Extracting constitutive parameters of the proposed unit cell. (a) The embedded SRR unit cell boundary. The dimensions are: $\mathrm{L}_{\text {cell }}=7.5, \mathrm{~W}_{\text {cell }}=3.3$, $\mathrm{w}_{\mathrm{s}}=0.1, \mathrm{~s}=1.1, \mathrm{~g}=0.1, \mathrm{l}_{\mathrm{c}}=6, \mathrm{~h}_{\mathrm{o}}=0.203, \mathrm{~h}_{\mathrm{m}}=0.813$ and the radius of vias $=0.1$, all in $\mathrm{mm}$. (b) Constitutive parameters of the proposed SRR.

corresponding SIW (w) is derived as,

$w_{e}=w-\frac{2 d^{2}}{0.95 p}$

Where $\mathrm{d}$ is the diameter of the vias and $\mathrm{p}$ is the center to center spacing between them. Here $\mathrm{d}=1 \mathrm{~mm}, \mathrm{p}=2.4 \mathrm{~mm}$ and a substrate of Rogers RO4003 with a relative permittivity of 3.55 and $\tan \delta=0.0027$ are used in all of our designs. The width of the equivalent rectangular waveguide at $f_{\text {cTEIO }}=7 \mathrm{GHz}$ is $w_{e}=1 /\left(2 f_{c T E_{10}} \sqrt{\varepsilon_{0} \varepsilon_{r} \mu_{0}}\right)=11.4 \mathrm{~mm}$. Substituting the above values for $\mathrm{d}, \mathrm{p}$ and $\mathrm{w}_{\mathrm{e}}$ in Eq. (1) results in $\mathrm{w}=12.2 \mathrm{~mm}$. Furthermore, an SIW-microstrip transition which is optimized for the best possible matching is shown in Fig. 1.

\section{B. The SRR structure}

Figure 2(a) shows a split ring resonator's unit cell representation. It is embedded in a thin dielectric substrate of dimensions $W_{\text {cell }} \times L_{\text {cell }}$. The top and bottom walls (xy planes)

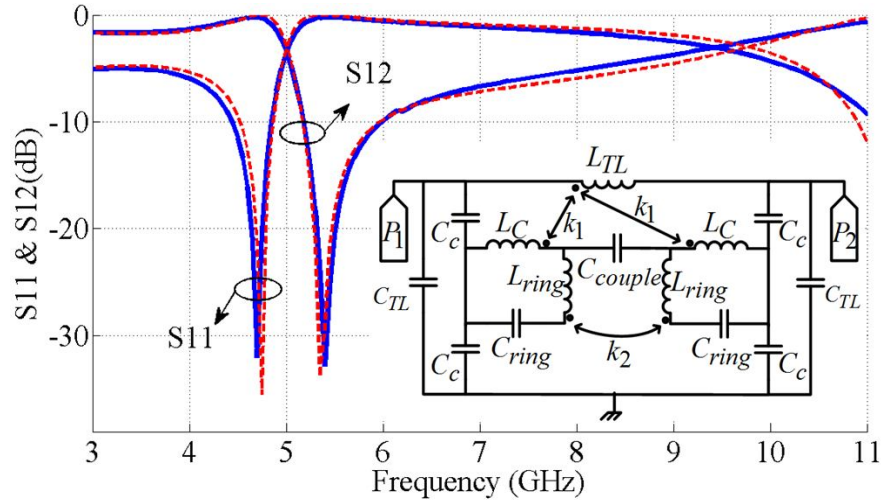

Fig. 3. The equivalent circuit modeling of the embedded SRR unit cell. Comparison of the equivalent circuit (dashed lines) with the HFSS results (solid lines). The element values for the unit cell of Fig. 2 are: $\mathrm{L}_{\mathrm{TL}}=0.66 \mathrm{nH}$, $\mathrm{C}_{\mathrm{TL}}=0.83 \mathrm{pF}, \quad \mathrm{L}_{\text {ring }}=1.06 \mathrm{nH}, \quad \mathrm{C}_{\text {ring }}=0.4 \mathrm{pF}, \quad \mathrm{L}_{\mathrm{C}}=0.86 \mathrm{nH} \quad \mathrm{C}_{\mathrm{c}}=0.2 \mathrm{pF}$, $\mathrm{C}_{\text {couple }}=0.4 \mathrm{pF}, \mathrm{k}_{1}=0.7, \mathrm{k}_{2}=0.58$

of the unit cell are prefect electric conductors (PEC), while the side walls ( $y z$ planes) are perfect magnetic conductors (PMC). $h_{o}$ and $h_{m}$ are the thicknesses of the middle, top or bottom sections, respectively. The boundary condition can hence support TEM wave propagation and the characteristics obtained from this unit cell can be generalized to the overall medium.

The unit cell is simulated using HFSS. Using the method proposed by Smith et al [5], the constitutive parameters of the unit cell can be obtained from the S-parameters of the unit cell. The real and imaginary parts of the $\varepsilon$ and $\mu$ of the unit cell are shown in Fig 2. (b).

The equivalent circuit model of the embedded SRR's unit cell is shown in the inset of Fig. 3. In this circuit $\mathrm{L}_{\mathrm{TL}}$ and $\mathrm{C}_{\mathrm{TL}}$ are the $\pi$-model elements of a transmission line environment which in this case is the substrate dielectric in which the SRRs are embedded. $\mathrm{L}_{\text {ring }}$ and $\mathrm{C}_{\text {ring }}$ are the equivalent inductance and capacitance of the SRRs. $\mathrm{k}_{1}$ is the coupling coefficient between the ring and the transmission line and $\mathrm{k}_{2}$ is the coupling coefficient between the rings of the two SRRs. $\mathrm{C}_{\text {couple }}$ and $\mathrm{C}_{\mathrm{c}}$ are coupling capacitances between the two SRRs and between the SRRs and dielectric substrate, respectively. The equivalent circuit model is analyzed using ADS and the results are compared with those of the unit cell in Fig. 3. As can be seen the two results are in good agreement.

\section{THE EFFECT OF LOADING SIW WITH SRRS}

An SRR-loaded rectangular waveguide can show uniaxial negative transversal permeability. The loaded structure can show backward-wave propagation. For this purpose the SRRs are etched on a dielectric slab and then the slab is placed in the magnetic wall of the waveguide [3]. But such a slab cannot be used in SIWs, since SIW structures are filled and the thickness of the substrate is not high enough.

Using the designed SIW and SRR structures presented in section II, a miniaturized SIW is realized which can easily be integrated with planar circuits. A double SRR unit cell embedded in the middle layer of the designed SIW is shown 
in Fig. 4 (a). The structure is composed of a layer with three sections; one with a thickness of $0.813 \mathrm{~mm}$ and the other two at top and bottom with $0.203 \mathrm{~mm}$ thickness. The substrate used is Rogers RO4003C ${ }^{\mathrm{TM}}$ with a dielectric constant of 3.55 and $\tan \delta=0.0027$.

The loaded SIW can be analyzed as follows. The SIW is modeled by an equivalent rectangular waveguide and then image theory is applied to obtain an infinite parallel plate waveguide (PPWG) containing the embedded SRRs, Fig. 4(b). On the other hand, the SRRs are excited by the components of the magnetic field which are perpendicular to their surface. Consequently, the relative permeability in these directions may be negative. However, the relative permeability in the directions where the components of the magnetic field are parallel to the SRRs is always positive and approximately equal to that of the vacuum. Thus in the dominate $\mathrm{TE}_{10}$ mode, $\mathrm{H}_{\mathrm{x}}$ is only perpendicular to the SRRs' surface, i.e. the permeability is uniaxial. Based on the above explanation and Fig. 4 (b), $\mu_{\mathrm{y}}=\mu_{\mathrm{z}}=\mu_{0}$ and $\mu_{\mathrm{x}}$ may be negative.

Hence the SRRs act as a uniaxial anisotropic material described by the following permeability tensor

$$
\bar{\mu}=\left[\begin{array}{ccc}
\mu_{x} & 0 & 0 \\
0 & \mu_{0} & 0 \\
0 & 0 & \mu_{0}
\end{array}\right]
$$

The propagation factor corresponding to the SIW structure loaded by the embedded SRRs can be determined in a similar procedure as given in [3]. The SRR-loaded SIW can simply be considered as a medium with a permeability tensor similar to (3). Solving the wave equations in such a medium the propagation factor for the lossless case can be obtained as

$\beta_{y}=-k_{0} \sqrt{\mu_{x(e f f)} \varepsilon_{r}\left(1-\left[\frac{f_{c}}{f}\right]^{2}\right)}$

Where $f_{c}=c /\left(2 w \sqrt{\varepsilon_{r} \mu_{r}}\right)$ and $\mu_{\mathrm{x}(\mathrm{eff})}$ is the permeability of a dispersive metamaterial,

$\mu_{x(e f f)}=1-\left(\frac{f_{m p}{ }^{2}-f_{\circ}^{2}}{f^{2}-f_{\circ}^{2}-j \gamma f}\right)$

Where $f$ is the operation frequency, $f_{m p}$ is the magnetic plasma frequency at which $\mu_{\mathrm{x}(\mathrm{eff})}=0, f_{0}$ is the resonant frequency and $\gamma$ represents losses.

In Eq. (4), when $\mu_{x(\text { eff })}$ is negative, backward waves can propagate below cutoff frequency $(f<f c)$, and a stop band is established above cutoff $(f>f c)$, [3].

Three kinds of SRRs with $f_{0}>f c, f_{0} \approx f_{c}$ and $f_{0}<f c$ have been designed and the corresponding transversal permeability $\left(\mu_{\mathrm{x}(\mathrm{eff})}=\mu_{\mathrm{x}(\mathrm{eff})}^{\prime}+\mathrm{j} \mu_{\mathrm{x}(\mathrm{eff})}\right)$ is sketched in Fig. 5. In all three cases, the parameters are the same as designed in section II for a

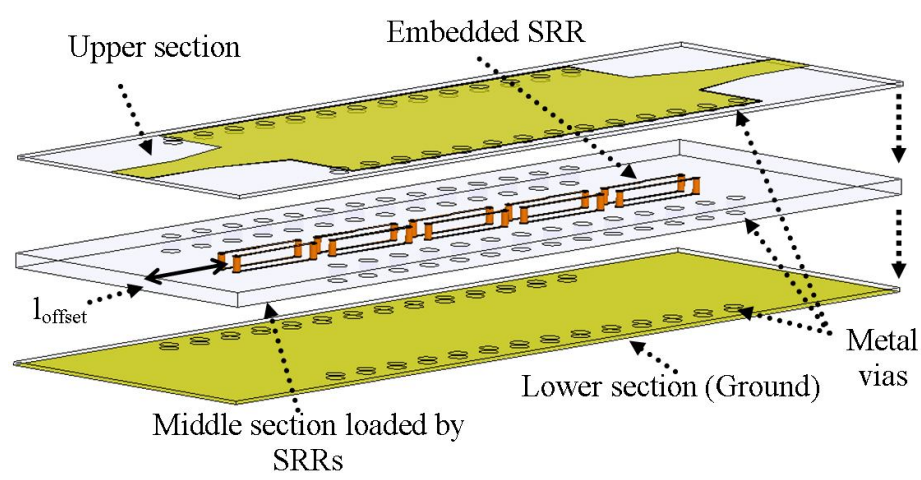

(a)

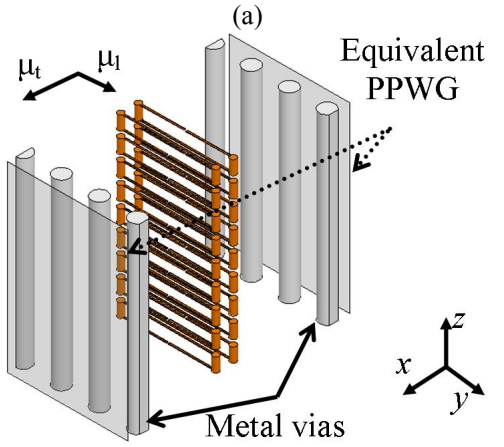

(b)

Fig. 4. The proposed structure. (a) SRR structure embedded in SIW, $1_{\text {offset }}=8 \mathrm{~mm}$ and the separation between the SRRs is $1.5 \mathrm{~mm}$. (b) Equivalent infinite parallel plate waveguide (PPWG) containing the SRRs.

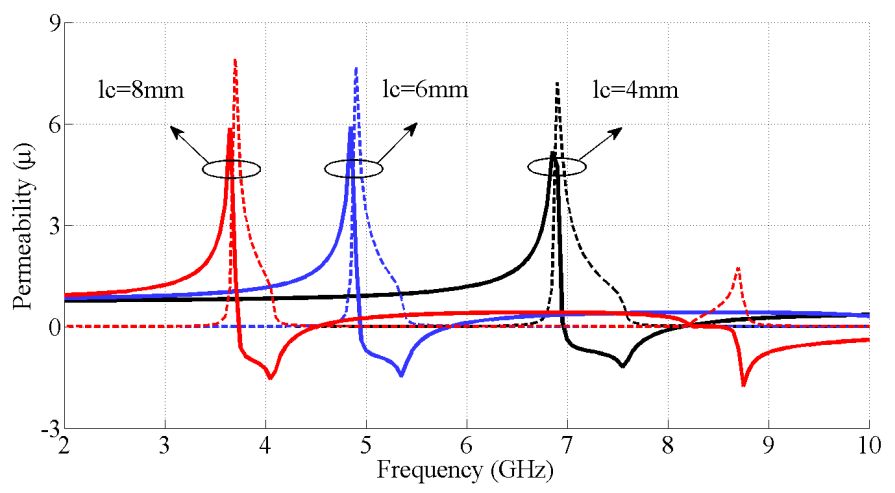

Fig. 5. Permeability constants for different values of $l_{c}, \mu^{\prime}$ (solid line) and $\mu^{\prime \prime}$ (dashed line).

unit cell (Fig. 2 (a)), except for $1_{c}$ which is variable and chosen to be 4, 6 and $8 \mathrm{~mm}$. The corresponding results for the unloaded and loaded SIW structure with the three proposed SRRs are illustrated in Fig. 6.

For $1_{\mathrm{c}}=4 \mathrm{~mm}$, a stopband is established in the transition waveguide region, which can be used for a band-stop filter with an excellent rejection band. This can also be seen from Fig. 5 which indicates that the relative permeability for $1_{\mathrm{c}}=4 \mathrm{~mm}$ is negative from $f_{0}$ to $f_{m p}$; i.e. from about 7-8.2 GHz. For $1_{c}=6 \mathrm{~mm}$, the permeability becomes negative close to $f_{c}$; therefore the passband for the backward waves is very close to the cutoff frequency. In this case the cutoff frequency begins very sharply at about $5.7 \mathrm{GHz}$, which is $1500 \mathrm{MHz}$ less than the cutoff frequency of the unloaded SIW, while the insertion loss in the worst case is only $-2.5 \mathrm{~dB}$. 


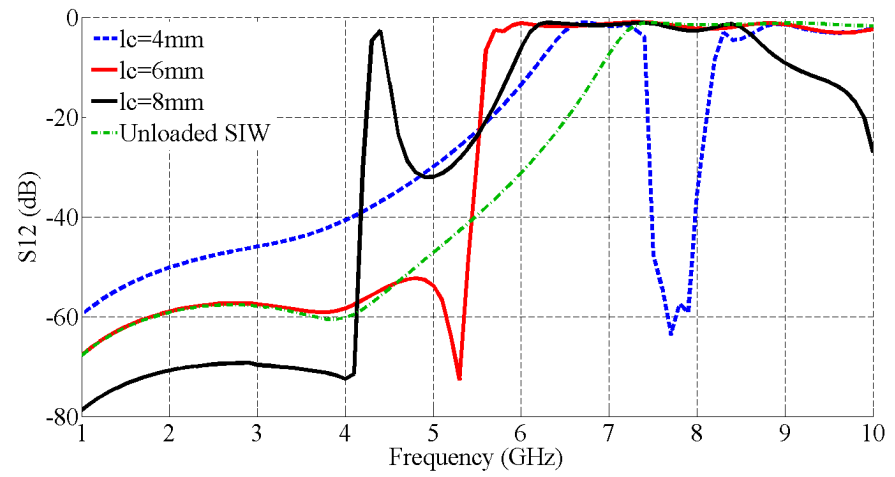

Fig. 6. Simulated S parameters for the SIW structure loaded by the proposed SRRs with three different lengths.

As shown in Fig. 6, when $1 \mathrm{c}=8 \mathrm{~mm}$, there are two negative region for the permeability; one at about $3.7-4.5 \mathrm{GHz}$ and the other above $8.5 \mathrm{GHz}$. Thus a passband for backward waves is formed before $f_{c}$ and a stopband is formed after $f_{c}$.

It should be noted that the passbands are formed when the permeability losses $\left(\mu^{\prime \prime}{ }_{x(\text { eff })}\right)$ are small; indicating to the reduction of bandwidth for the backward-wave passbands. In all the three cases discussed, the loaded SIW shows a reduction of about $1.3-1.5 \mathrm{GHz}$ in cutoff frequency compared to the unloaded SIW due to an increase in the electric permittivity, Fig. 2 (b).

Based on the three above cases, the SSRs can be designed arbitrarily; thus further miniaturization is also possible; i.e. the width of the loaded SIW can be less than half of the wavelength at cutoff frequency $\left(\lambda_{\mathrm{c}}\right)$.

A simple method to prove the existence of backward-wave propagation is to measure the phase of S21 between two different physical lengths of the loaded SIW $\left(\mathrm{L}_{1} \& \mathrm{~L}_{2}\right.$ where $\left.\mathrm{L}_{1}>\mathrm{L}_{2}\right)$. In backward-wave passband $\varphi_{\mathrm{S} 12}\left(\mathrm{~L}_{2}\right)-\varphi_{\mathrm{S} 12}\left(\mathrm{~L}_{1}\right)>0$, [3]. This is shown in Fig. 7 for two different physical lengths of the loaded SIW in backward-wave propagation region.

The proposed structure for $1_{c}=8 \mathrm{~mm}$ is fabricated and tested. The diameters of the SRRs' vias are $0.4 \mathrm{~mm}$ instead of $0.2 \mathrm{~mm}$ used in simulations due to a limitation in the fabrication process. The simulated and measured results are shown in Fig. 8. The passband for the backward waves is formed at $4.75 \mathrm{GHz}$. Although the circuit is handmade, the two results are in good agreement.

\section{CONCLUSION}

In this paper, embedded SRRs are employed to provide negative uniaxial magnetic permeability. The SRRs are analyzed theoretically. An SIW structure is designed and loaded by the embedded SRRs and it is shown that it can support propagation backward-wave below cutoff frequency. Also, convenient designs can be adopted to obtain different passbands for backward waves below the cutoff frequency and to provide excellent filtering functionality above cutoff. This structure can be used in compact and planar microwave and

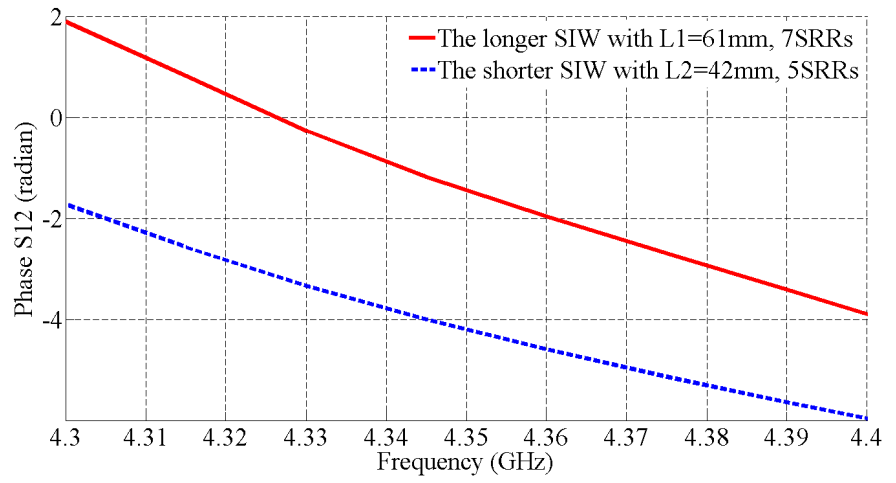

Fig. 7. Phase difference comparison to prove existence of back-ward wave propagation for the structure with $1_{\mathrm{c}}=8 \mathrm{~mm}$

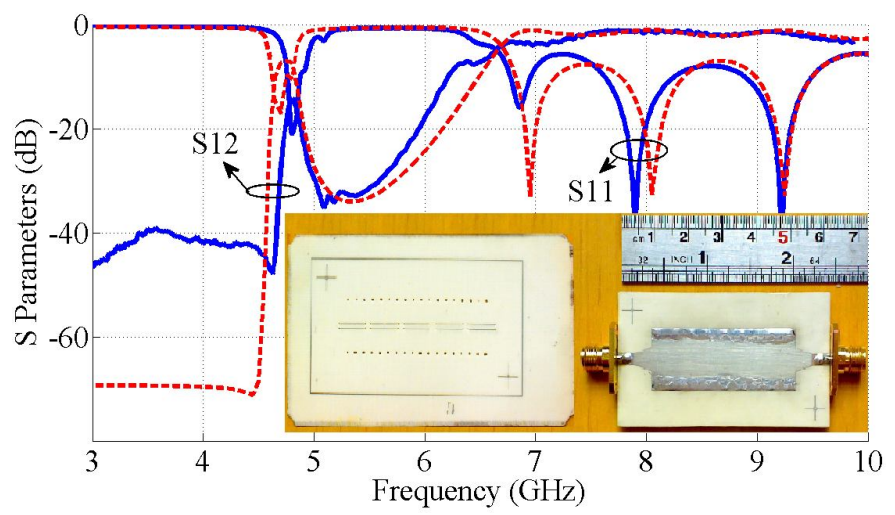

Fig. 8. Measured (solid lines) and simulated (dashed lines) $\mathrm{S}$ parameters for the loaded SIW with $1_{\mathrm{c}}=8 \mathrm{~mm}$ and radius of the SRRs' vias of $0.4 \mathrm{~mm}$. The fabricated circuit is shown in the bottom. the middle section is shown in the left.

millimeter wave components. Finally, the loaded structure was fabricated and the measured results demonstrated that the structure performs well and fulfills our expectations.

\section{REFERENCES}

[1] Y. Cassivi, L. Perregrini, P. Arcioni, M. Bressan, K. Wu, and G. Conciauro, "Dispersion characteristics of substrate integrated rectangular waveguide," Microwave and Wireless Components Letters, IEEE, vol. 12, pp. 333-335, 2002.

[2] Bozzi, M.; Feng Xu; Deslandes, D.; Ke Wu; , "Modeling and Design Considerations for Substrate Integrated Waveguide Circuits and Components," Telecommunications in Modern Satellite, Cable and Broadcasting Services, 2007. TELSIKS 2007. 8th International Conference on, vol., no., pp.P-VII-P-XVI, 26-28 Sept. 2007

[3] S. Hrabar, J. Bartolic, and Z. Sipus, "Waveguide miniaturization using uniaxial negative permeability metamaterial," Antennas and Propagation, IEEE Transactions on, vol. 53, pp. 110-119, 2005.

[4] D. Deslandes and K. Wu, "Accurate modeling, wave mechanisms, and design considerations of a substrate integrated waveguide," Microwave Theory and Techniques, IEEE Transactions on, vol. 54, pp. 2516-2526, 2006.

[5] D. Smith, D. Vier, T. Koschny, and C. Soukoulis, "Electromagnetic parameter retrieval from inhomogeneous metamaterials," Physical Review E, vol. 71, p. 36617, 2005. 